\title{
A CHARACTERIZATION OF SEMIPRIME IDEALS IN NEAR-RINGS
}

\section{SAMBASIVA RAO}

(Received 3 December 1980; revised 4 March 1981)

Communicated by R. Lidl

\begin{abstract}
It is well known that in any near-ring, any intersection of prime ideals is a semiprime ideal. The aim of this paper is to prove that any semiprime ideal $I$ in a near-ring $N$ is the intersection of all minimal prime ideals of $I$ in $N$. As a consequence of this we have any seimprime ideal $I$ is the intersection of all prime ideals containing $I$.
\end{abstract}

1980 Mathematics subject classification (Amer. Math. Soc.): primary 16 A 76; secondary 08 A 40.

\section{Preliminaries}

A near-ring is an algebraic system $(N,+, \cdot)$ satisfying (i) $(N,+)$ is a group, (ii) $(N, \cdot)$ is a semigroup and (iii) $(x+y) z=x z+y z$ for all $x, y, z$ in $N$. We abbreviate $(N,+, \cdot)$ by $N$.

If $S$ and $T$ are subsets of $N$, we denote the set $\{s t \mid s \in S, t \in T\}$ by $S T$. A normal subgroup $I$ of $(N,+)$ is called an ideal of $N(I \unlhd N)$ if $I N \subseteq I$ and for all $n, n^{\prime} \in N$ and for all $i \in I, n\left(n^{\prime}+i\right)-m n^{\prime} \in I$. An ideal $P$ of $N$ is called a prime ideal if for any ideals $I$ and $J$ of $N, I J \subseteq P$ implies either $I \subseteq P$ or $J \subseteq P$. An ideal $I$ of $N$ is called a semiprime ideal if for any ideal $J$ of $N, J^{2} \subseteq I$ implies that $J \subseteq I$. An ideal minimal in the set of all prime ideals containing some given ideal $I$ is called a minimal prime ideal of $I$ in $N$.

If $x$ is an element of $N$, then the principal ideal generated by $x$ is denoted by (x). If $S$ is a subset of $N$, we write $N \backslash S=\{n \in N \mid n \notin S\}$. A subset $M$ of a near-ring $N$ is called an $m$-system if for any $a, b \in M$ there exists $a_{1} \in(a)$ and

(1) Copyright Australian Mathematical Society 1982 
$b_{1} \in(b)$ such that $a_{1} b_{1} \in M$. A subset $S$ of a near-ring $N$ is called an $S p$-system if for any $s \in S$, there exists $s_{1} \in(s)$ and $s_{2} \in(s)$ such that $s_{1} s_{2} \in S$.

\section{2.}

In this section we prove the main theorem. Before proving this theorem, we state a lemma.

LEMMA 2.1. Let $I$ be an ideal in a near-ring $N$ and $M$ be an $m$-system in $N$ such that I $\cap M$ is empty. Then

(i) There is an $m$-system $M^{*}$ maximal relative to the properties: $M \subseteq M^{*}$, $I \cap M^{*}$ is empty.

(ii) If $M^{*}$ is an m-system maximal relative to the properties: $M \subseteq M^{*}, I \cap M^{*}$ is empty, then $N \backslash M^{*}$ is a minimal prime ideal of $I$ in $N$.

Proof. Immediate from 2.75, 2.80 and 2.81 of G. Pilz (1977).

Now we prove the main theorem.

THEOREM 2.2. If $I$ is any semiprime ideal in a near-ring $N$, then $I$ is the intersection of all minimal prime ideals of $I$ in $N$.

Proof. Let $I$ be any semiprime ideal of $N$. Then by 2.89(b) of G. Pilz (1977), $N \backslash I$ is an $S p$-system. So by 2.92 of G. Pilz (1977), we have that for each $s \in N \backslash I$ there exists an $m$-system $M$ in $N$ such that $s \in M \subseteq N \backslash I$. So $M \cap I$ is empty. Now by the above lemma, there exists a minimal prime ideal $P$ of $I$ such that $P \cap M$ is empty. But $s \in M$. Hence $s \notin P$. Therefore $I=\cap_{P \supset I} P$, where $P$ ranges over all minimal prime ideals of $I$. Thus every semiprime ideal $I$ is the intersection of all minimal prime ideals of $I$.

COROLlary 2.3. If $I$ is a semiprime ideal in a near-ring $N$, then $I$ is the intersection of all prime ideals containing $I$.

Proof. By the above theorem, $I=\cap P$, where $P$ ranges over all minimal prime ideals of $I, \supseteq \cap_{P \supset I} P$, where $P$ ranges over all prime ideals containing $I$. Therefore $I$ is the intesection of all prime ideals containing $I$.

I wish to acknowledge my thanks to Dr. Y. Venkateswara Reddy, my Research Director for giving me this problem and valuable suggestions in the preparation of this paper. 


\section{References}

G. Pilz (1977), Near-rings (North-Holland, Amsterdam-New York).

\section{Department of Mathematics}

Nagarjuna University

Nagarjunanagar-522 510
A. P. India 